\section{Glucan in clinical trials}

\section{Editorial}

Preliminary experiments on animal cancer models were so promising it is no wonder that these experiments are being repeated in several clinical trials. Additional clinical trials focusing on soluble glucan Imprime $\mathrm{PGG}^{\mathrm{TM}}$ in combination with monoclonal antibody from ImClone System, Erbitux ${ }^{\mathrm{TM}}\left(\right.$ Cetuximab $\left.^{\mathrm{TM}}\right)$, and Irinotecan ${ }^{\mathrm{TM}}$ are currently under way. As the preliminary results are already exceeding all expectations, the manufacturer of Imprime $\mathrm{PGG}^{\mathrm{TM}}$, Biothera, has already started additional cancer clinical trials. Based on latest information, current Phase II clinical lung cancer trials have achieved stage one endpoints, demonstrating a significant level of efficacy among the initial group of patients. The improvements reached up to $48 \%$ and in biomarker-positive patients were results even more impressive- $67 \%$ improvement. These studies, which will enroll up to 90 patients each, support the synergy of glucan and monoclonal antibody therapies. In addition, Biothera is conducting a Phase III clinical trial evaluating the combination therapy of Imprime $\mathrm{PGG}^{\mathrm{TM}}$ and Erbitux ${ }^{\mathrm{TM}}$ in colorectal cancer patients. This study is currently enrolling up to 795 patients and will be conducted at 50 locations worldwide, including the U.S, France and Germany. Results achieved so far are highly promising showing the response rate for patients receiving glucan nearly doubled and median progression-free survival time increased $41 \%$. In addition, Biothera announced that Imprime $\mathrm{PGG}^{\mathrm{TM}}$ had achieved significant results in meeting endpoint results in the Phase IIb NSCLC clinical trial. Patients in this trial had an objective response rate more than double compared to the control group. In the biomarker-positive group, the results were even better. In case of chronic lymphocytic leukemia, a high number of patients with a complete response after Imprime and antibody combination is extremely impressive. It is even more impressive when we remember that today most cancer drugs have a response rate well below $40 \%$.

Additional humanized antitumor monoclonal antibodies (Rituximab $^{\mathrm{TM}}$ and Campath-1H) are promising targets. Clearly, glucan functions as a potent adjuvant for monoclonal antibody therapy of cancer to elicit novel neutrophil and macrophage-mediated tumor-killing mechanisms that are not activated by monoclonal antibody therapy alone. Even when glucan by itself was repeatedly shown to cause tumor regression, it was most probably a result of cooperation with natural antitumor antibodies. However, combined therapy with monoclonal antibodies or vaccines in combination with glucan offers several advantages: it is easier to elicit an antibody response than cytotoxic lymphocytes, and the therapy can use humanized antibodies and therefore not rely on the patient's own immune response that is frequently suppressed because of tumor burden and previous irradiation or chemotherapy. The preliminary results are often so promising that glucan-producing companies are acquiring anti-cancer monoclonal antibodies from their developers. More details on the current use of glucan in clinical trials are given in a great review. ${ }^{1}$ Additional trials studied mushroom-derived glucan Maitake in myelodysplastic syndromes. This disease is characterized by ineffective erythropoiesis with dysplastic bone marrow leading to peripheral cytopenia, risk of infections and progression to acute myelogenous leukemia. The study showed increased neutrophils and monocyte functions. ${ }^{2}$ Readers seeking more information about glucan
Volume 5 Issue 3 - 2017

\author{
Vetvicka V,Vetvickova J \\ Department of Pathology, University of Louisville, USA
}

Correspondence: Vaclav Vetvicka, University of Louisville, Department of Pathology, Louisville, KY 40202, USA, Fax 502852-I I77, Email vaclav.vetvicka@louisville.edu

Received: November 23, 2017 | Published: November 28, 2017

and their application in cancer therapy, with particular attention of human clinical trials, should read an excellent review by Aleem. ${ }^{3}$

In our research, we focused on children with respiratory problems. Upper respiratory infections have the highest incidence of acute illness in the developed world. There is no doubt that a need for a safe, effective, inexpensive treatment for chronic respiratory problems exists. Using a long-term cooperation with Sanatorium for Respiratory Disease EDEL, which is located in North Moravia in an area of extremely low air pollution, we conducted a series of clinical, placebo-driven trials of the effects of a 30day supplementation with Glucan \#300 on immune parameters in children with chronic respiratory problems. The sanatorium offers not only optimal therapeutic treatment, but also a complexity of additional treatments, including speleotherapy. As a part of long-term observation, randomly selected groups of children with respiratory problems were treated with $100 \mathrm{mg}$ of orally administered glucan. Several parameters were evaluated at the beginning and at the end of the study. Three parameters, salivary calprotectin, albumin and C-reactive protein were used for the evaluation of acute inflammatory response and the quality of the nonspecific immunity of children. Our data showed the strong effects of glucan supplementation on all three tested parameters.

In addition to acute inflammatory response, we also studied the effects on formation of salivary antibodies. Our data demonstrateed that at the beginning of the study, the levels of antibodies were comparable in both groups. However, at the end of the study we found an increase of all three types of antibodies only in the glucan-treated group, whereas the placebo group expressed no changes or even a decrease in antibody production. In addition, glucan-supplemented children showed increase in physical endurance and an overall health. ${ }^{4}$ These findings represent just another stone in the mosaic of glucan-mediated response in disease prone-children. From these data, we can conclude that even a relatively short supplementation of food with glucan has positive stimulatory effects on mucosal immunity. Based on our data, glucan administration might be considered a highly promising and inexpensive method in the treatment of chronic respiratory problems in children. In addition, our study strongly suggested that oral administration of glucan has positive effects in patients suffering from benign prostate hyperplasia.

\section{Acknowledgements}

None. 


\section{Conflicts of interest}

The Editor declares there are no conflicts of interest.

\section{References}

1. Yan J. Biology and Chemistry of Beta Glucan Vol: 1 Beta GlucansMechanisms of action. In: Vaclav Vetvicka, et al. editors. Benthama Science. UAE; 2011. p. 39-47.
2. Wesa KM, Cunningham Rundles S, Klimek VM, et al. Maitake mushroom extract in myelodysplastic syndromes (MDS): a phase II study. Cancer Immunol Immunothe. 2015;64(2):237-247.

3. Aleem E. $\boldsymbol{\beta}$-Glucans and their applications in cancer therapy: focus on human studies. Anticancer Agents Med Chem. 2013;13(5):709-719.

4. Vetvicka V, Rcihter J, Svozil V, et al. Placebo-driven clinical trials of yeastderived $\boldsymbol{\beta}$-(1-3) glucan in children with chronic respiratory problems. Ann Transl Med. 2013;1(3):26. 